\title{
ON SETS NONMEASURABLE WITH RESPECT TO INVARIANT MEASURES
}

\author{
SLAWOMIR SOLECKI
}

(Communicated by Andrew M. Bruckner)

\begin{abstract}
A group $G$ acts on a set $X$, and $\mu$ is a $G$-invariant measure on $X$. Under certain assumptions on the action of $G$ and on $\mu$ (e.g., $G$ acts freely and is uncountable, and $\mu$ is $\sigma$-finite), we prove that each set of positive $\mu$-measure contains a subset nonmeasurable with respect to any invariant extensions of $\mu$. We study the case of ergodic measures in greater detail.
\end{abstract}

\section{INTRODUCTION}

The nonmeasurability of the classical Vitali's example of a Lebesgue nonmeasurable set on the real line relies first of all on the invariance of the Lebesgue measure. One can successfully and without any changes apply this construction to a finite invariant measure on an abstract infinite group. This indicates that a problem of finding a nonmeasurable set for an invariant measure may be of a different nature than the general measure problem (the problem of finding a nonmeasurable set for any, not necessarily invariant, diffused measure, i.e., disproving the existence of real-valued measurable cardinals); however, Zakrzewski in [9] and, earlier in the Abelian case, Pelc (see [7, Theorem 2.5]) constructed a universal diffused semifinite invariant measure on any group of cardinality not less than the first real-valued measurable cardinal. Nevertheless one may still hope that some purely measure-theoretic properties of the Lebesgue measure on the real line can be found, so for each invariant measure enjoying them there exists a nonmeasurable set. Obviously these conditions have to be stronger than semifiniteness.

In this direction Harazišvili in [6] and independently Erdös and Mauldin in [4] proved that for any $\sigma$-finite invariant measure on an uncountable group there exists a nonmeasurable set. Harazišvili observed that any set of positive measure contains such a nonmeasurable set. Their arguments are still connected with the general measure problem as they use Ulam's theorem that $\omega_{1}$ is not real-valued measurable. Ryll-Nardzewski and Telgársky in [8] found a nice refinement of this result also using Ulam's method. Pelc in [7, p. 15] asked about a generalization of Harazišvili's and Erdős and Mauldin's theorem: "Given a $\sigma$-finite

Received by the editors January 13, 1992.

1991 Mathematics Subject Classification. Primary 28C10; Secondary 43A05.

Key words and phrases. Invariant measures, nonmeasurable sets, extensions of measures. 
invariant measure $m$ on a group $G$, does every set of positive measure contain a subset nonmeasurable with respect to any invariant extension of $m$ ?" This question is motivated by the fact that Vitali's example is nonmeasurable with respect to any invariant extension of the Lebesgue measure. Pelc [7, Proposition 2.3 $\left(^{1}\right)$ ] answered the question in the affirmative if $m$ is an invariant extension of a regular $\sigma$-finite Haar measure on a topological group. Previously, Harazišvili had noticed it for $m$ an invariant extension of the Lebesgue measure.

In the present paper we give an affirmative answer to Pelc's question in full generality. Our proof being free of Ulam's method shows that the nonexistence of universal measures on small cardinals has little to do with the nonexistence of universal $\sigma$-finite invariant measures on groups. We extract also two other properties of the Lebesgue measure on the $n$-dimensional Euclidean space under which the above question has an affirmative answer: if $L^{1}$ has a dense subset of cardinality less than the cardinality of the group or if the measure is ergodic and nonatomic. In fact, we give sufficient and necessary conditions for ergodic measures ensuring that the answer is affirmative.

The author thanks Piotr Zakrzewski for helpful remarks.

\section{Conventions}

Throughout the paper we assume that we are given a set $X$ and a group $G$ acting on $X$. We write $H Y=\{h x: x \in Y, h \in H\}$ for $H \subset G, Y \subset X$ and $h Y=\{h\} Y$ for $h \in G, Y \subset X$.

A measure $\mu$ defined on a $\sigma$-algebra of subsets of $X$ (called the $\sigma$-algebra of $\mu$-measurable sets) is said to be invariant if $h Y$ is $\mu$-measurable for $\mu$ measurable $Y$ and $h \in G$ and $\mu(h Y)=\mu(Y)$. Where confusion cannot occur we say measurable rather than $\mu$-measurable. Writing $\mu(A)$ we implicitly assume that $A$ is $\mu$-measurable. For any $A \subset X$ let $\mu_{*}(A)=\sup \{\mu(B): B$ is $\mu$-measurable and $B \subset A\}$ and $\mu^{*}(A)=\inf \{\mu(B): B$ is $\mu$-measurable and $B \supset A\}$. If $A$ is a $\mu$-measurable set, by $\mu \mid A$ we denote the restriction of $\mu$ to the family of all measurable sets included in $A$. The action of $G$ is $\mu$-free if $\mu^{*}(\{x \in X: h x=x\})=0$ for any $h \in G \backslash\{e\} \quad(e=$ the identity of $G)$. Notice that the action of any subgroup of the group of all isometries of a Euclidean space is $\mu$-free for any invariant extension of the Lebesgue measure.

Our convention is similar to that assumed in [6] and a little more general than that normally assumed when one considers a group with a left-invariant measure on it (as in Pelc's question). What we assume seems to be more natural when one thinks of some groups of isometries acting on $R^{n}$ and, on the other hand, it encompasses the case when the measure on a group is left invariant only with respect to some subgroup as, e.g., in [7, Theorem 2.2].

A measure $\bar{\mu}$ is called an invariant extension of $\mu$ if $\bar{\mu}$ is an invariant measure, each $\mu$-measurable set is $\bar{\mu}$-measurable, and $\mu(Y)=\bar{\mu}(Y)$ for any $\mu$ measurable set $Y \subset X$. An invariant extension $\bar{\mu}$ of $\mu$ is localizable if for any $\bar{\mu}$-measurable set $Y \subset X$ with $\bar{\mu}(Y)>0$, there is a $\mu$-measurable set $Y_{1} \subset X$ such that $\mu\left(Y_{1}\right)<\infty$ and $\bar{\mu}\left(Y \cap Y_{1}\right)>0$. We see that if a measure $\mu$ has a localizable extension, then $\mu$ is semifinite, i.e., each set of positive measure contains a set of positive and finite measure. Measures of this kind are called semiregular in [7,9]. Let us notice that a measure is semifinite if and only if it is 
a localizable extension of itself. A measure $\mu$ on $X$ is $\sigma$-finite if $X=\bigcup_{n=1}^{\infty} X_{n}$ where $X_{n}$ are $\mu$-measurable with $\mu\left(X_{n}\right)<\infty$. It is clear that each extension of a $\sigma$-finite measure is localizable. An invariant measure $\mu$ is called ergodic if for any two measurable sets $A, B \subset X$ with $\mu(A)>0$ and $\mu(B)>0$ there is an $h \in G$ such that $\mu(A \cap h B)>0$.

Let $\mu$ be an invariant measure on $X$. A set $A$ is called infinitely covered by $H \subset G$ if $H$ is countably infinite and there is a $\mu$-measurable set $B$ of finite measure such that each element of $A$ belongs to infinitely many sets from $\{h B: h \in H\}$, i.e., $A \subset \bigcap_{m=1}^{\infty} \bigcup_{n>m} h_{n} B$ if $H=\left\{h_{n}: n \in N\right\}, h_{n} \neq h_{m}$ for $n \neq m$. We say that a set is infinitely covered if it is infinitely covered by some countably infinite $H \subset G$. A set $V \subset X$ is called a Vitali set of a subgroup $H$ of $G$ if $V \cap H\{x\}$ has exactly one element for any $x \in X$. Such sets are sometimes called $H$-selectors.

All measures considered in this paper are assumed to attain at least one positive value. We call a measure nontrivial if it attains at least one positive and finite value.

$|A|$ denotes the cardinality of $A . A \triangle B$ denotes the symmetric difference of $A$ and $B$, i.e., $A \triangle B=(A \backslash B) \cup(B \backslash A) . N$ stands for the set of positive integers.

\section{SMall measures}

Throughout this section we assume that the action of $G$ is $\mu$-free for an invariant measure $\mu$.

Lemma 3.1. Assume the action of $G$ is $\mu$-free. Let $A$ be infinitely covered by $H$. If $V$ is a Vitali set of a subgroup of $G$ containing $H$, then $\bar{\mu}_{*}(V \cap A)=0$ for any invariant extension $\bar{\mu}$ of $\mu$.

Proof. Let $H=\left\{h_{n}: n \in N\right\}$ with $h_{n} \neq h_{m}$ for $n \neq m$. Denote by $H^{\prime}$ the group containing $H$ of which $V$ is a Vitali set. Let $B$ be a set of finite $\mu$-measure such that $A \subset \bigcap_{m=1}^{\infty} \bigcup_{n>m} h_{n} B$, and let $A^{\prime}$ be any $\bar{\mu}$-measurable set contained in $V \cap A$. Put $V_{n}=A^{\prime} \cap h_{n} B$. Then $\bigcup_{n=1}^{\infty} h_{n}^{-1} V_{n} \subset B$. If $h_{m} h_{n}^{-1} v \in V_{m}$ for some $v \in V_{n}$ and $m \neq n$, then, in fact, $h_{m} h_{n}^{-1} v=v$ since distinct elements of $V$ belong to different orbits of $H^{\prime}$. Thus $h_{n}^{-1} V_{n} \cap$ $h_{m}^{-1} V_{m} \subset\left\{x \in X: h_{m} h_{n}^{-1} x=x\right\}$. Since the action of $G$ is $\mu$-free, we have $\mu^{*}\left(h_{n}^{-1} V_{n} \cap h_{m}^{-1} V_{m}\right)=0$; therefore, we obtain

$$
\infty>\mu(B) \geq \sum_{n=1}^{\infty} \bar{\mu}\left(h_{n}^{-1} V_{n}\right)=\sum_{n=1}^{\infty} \bar{\mu}\left(V_{n}\right)
$$

Then

$$
0=\lim _{m} \sum_{n=m}^{\infty} \bar{\mu}\left(V_{n}\right) \geq \bar{\mu}\left(\bigcap_{m=1}^{\infty} \bigcup_{n>m} V_{n}\right)=\bar{\mu}\left(A^{\prime} \cap \bigcap_{m=1}^{\infty} \bigcup_{n>m} h_{n} B\right)=\bar{\mu}\left(A^{\prime}\right) .
$$

Lemma 3.2. Let $A$ contain a set of positive $\mu$-measure infinitely covered by $H$. Then there exists a Vitali set $V$ for the group generated by $H$ such that $A \cap V$ is nonmeasurable with respect to any invariant extension of $\mu$.

Proof. Let $A^{\prime}$ be a subset of $A$ infinitely covered by $H$ with $\mu\left(A^{\prime}\right)>0$. Let $H^{\prime}$ be the subgroup generated by $H$, and let $\Xi$ be the family of all orbits of 
$H^{\prime}$ intersecting $A^{\prime}$. Let $V^{0}$ be a set contained in $A^{\prime}$, selecting one element from each member of $\Xi$. One can easily extend $V^{0}$ to a Vitali set $V$.

Assume that there is $\bar{\mu}$, which is an invariant extension of $\mu$ and for which $A \cap V$ is measurable. Then $A \cap V \cap A^{\prime}=V \cap A^{\prime}=V^{0}$ is $\bar{\mu}$-measurable, too. Since $A^{\prime} \subset H^{\prime} V^{0}$ and $H^{\prime}$ is countable, we have $\bar{\mu}\left(V^{0}\right)>0$, which contradicts Lemma 3.1.

Lemma 3.3. Assume $G$ is uncountable. Let $\mu$ be $\sigma$-finite and let $\mu(A)>0$. Then $A$ contains an infinitely covered set of positive measure.

Proof. Since $\mu$ is $\sigma$-finite, $A$ contains a measurable set $A^{\prime}$ of positive and finite measure. Let $X=\bigcup_{k=1}^{\infty} X_{k}$ where the $X_{k}$ are measurable with $\mu\left(X_{k}\right)<$ $\infty$, and let

$$
G_{k}=\left\{h \in G: \mu\left(h X_{k} \cap A^{\prime}\right)>0\right\} .
$$

If all the $G_{k}$ 's are countable, pick $h \in G \backslash \bigcup_{k=1}^{\infty} G_{k}$. Then

$$
0<\mu\left(A^{\prime}\right)=\mu\left(h X \cap A^{\prime}\right)=\mu\left(\bigcup_{k=1}^{\infty} h X_{k} \cap A^{\prime}\right) \leq \sum_{k=1}^{\infty} \mu\left(h X_{k} \cap A^{\prime}\right)=0,
$$

which is a contradiction. Thus for some $k \in N$ the set $G_{k}$ is uncountable. We can find $\varepsilon>0$ such that $\mu\left(h X_{k} \cap A^{\prime}\right)>\varepsilon$ for infinitely many $h$, say for $h_{n}$, $n \in N \quad\left(h_{n} \neq h_{m}\right.$ if $\left.n \neq m\right)$. Then

$$
\mu\left(A^{\prime} \cap \bigcap_{m=1}^{\infty} \bigcup_{n>m} h_{n} X_{k}\right)=\inf _{m \in N} \mu\left(A^{\prime} \cap \bigcup_{n>m} h_{n} X_{k}\right) \geq \varepsilon>0 .
$$

For an uncountable $G$ a special case $(X=G)$ of the following theorem gives the answer to the question of Pelc mentioned in the introduction. In the case when $G$ is countable the answer is straightforward; namely, each invariant measure on $G$ is equal to the counting measure up to a multiplicative constant restricted to an invariant $\sigma$-algebra. Each such measure can be extended in the obvious way to a measure defined on the $\sigma$-algebra of all subsets of $G$, so the answer is negative; but if we take into account only diffused measures, as in [7], then the answer is in the affirmative because there are no such invariant measures on $G$.

Theorem 3.1. Let $\mu$ be $\sigma$-finite and invariant, and let $G$ be uncountable and act $\mu$-freely. Then each set of positive measure contains a subset nonmeasurable with respect to any invariant extension of $\mu$.

Proof. This is an immediate consequence of Lemmas 3.3 and 3.2.

For a measure $m$ on $X$ we denote by $L^{1}(m)$ the space of equivalence classes of summable (with respect to $m$ ) functions on $X$ with the usual norm $\|f\|_{1}=\int_{X}|f| d m$. This norm induces a metric, so $L^{1}(m)$ may be considered a metric space. The following lemma, useful in proving Theorem 3.2, does not use the invariance of $m$.

Lemma 3.4. Let $A_{i}, i \in I$, be a family of measurable sets with $a \leq m\left(A_{i}\right)<\infty$ for some $a>0$ indexed (perhaps with repetitions) by a set $I$. Let $L^{1}(m)$ have a dense subset of cardinality less than $|I|$. Then for any $\varepsilon>0$ there exists an infinite set $\left\{i_{n}: n \in N\right\} \subset I$ such that $m\left(\bigcap_{n=1}^{\infty} A_{i_{n}}\right) \geq a-\varepsilon$. 
Proof. Let $\chi_{i} \subset L^{1}(m)$ be the characteristic function of $A_{i}, i \in I$. Since $L^{1}(m)$ is a metric space possessing a dense subset of cardinality less than $|I|$, each discrete subset of $L^{1}(m)$ has cardinality less than $|I|$ (see [3, Theorem 4.1 .15$, p. 318]). Thus, again because $L^{1}(m)$ is metric, there is a sequence $\chi_{i_{n}}, n \in N$, with $i_{n} \neq i_{m}$ for $n \neq m$, which converges in $L^{1}(m)$ to some function $f$. Since we can choose a subsequence of $\chi_{i_{n}}$ which converges to $f$ pointwise and also since $m\left(A_{i_{n}}\right)=\left\|\chi_{i_{n}}\right\|_{1}$ tend to $\|f\|_{1}$, we can assume that $f$ is a characteristic function of a measurable set $A$ with $m(A) \geq a$. Choose a subsequence $\chi_{i_{n_{k}}}, n_{k_{1}} \neq n_{k_{2}}$ for $k_{1} \neq k_{2}$, such that $\left\|f-\chi_{i_{n_{k}}}\right\|_{1} \leq \varepsilon / 2^{k}$. Then $m\left(A \backslash A_{i_{n_{k}}}\right) \leq \varepsilon / 2^{k}$, whence $m\left(A \backslash \bigcap_{k=1}^{\infty} A_{i_{n_{k}}}\right) \leq \varepsilon$; thus $m\left(\bigcap_{k=1}^{\infty} A_{i_{n_{k}}}\right) \geq$ $a-\varepsilon$.

The $\sigma$-finiteness of $m$ is implied, at least when $m$ is semifinite, by the separability of $L^{1}(m)$. The following theorem shows that the countability assumption in the second case may be relaxed.

Theorem 3.2. Let $m$ be an invariant measure on $X$. Suppose $L^{1}(m)$ has a dense subset of cardinality less than $|G|$. Let $\mu$ be an invariant localizable extension of $m$. Assume that $G$ acts $\mu$-freely. Then each set of positive $\mu$ measure contains a subset nonmeasurable with respect to any invariant extension of $\mu$.

Proof. Let $A$ be such that $\mu(A)>0$. Find $B$ with $\infty>m(B)=a$ and $\mu(A \cap B)>0$. Let $\mu(A \cap B)>2 \varepsilon>0$, and consider the family $g B, g \in G$. By Lemma 3.4 we can find a set $\left\{g_{n}: n \in N\right\} \subset G$ with $g_{n} \neq g_{m}$ for $n \neq m$ such that $m\left(\bigcap_{n=1}^{\infty} g_{n} B\right) \geq a-\varepsilon$. Put $h_{n}=g_{1}^{-1} g_{n}$. Then, for each $n \in N$, $\mu\left(B \cap \bigcap_{n=1}^{\infty} h_{n} B\right) \geq a-\varepsilon ;$ thus,

$$
\begin{aligned}
\mu\left(A \cap \bigcap_{m=1}^{\infty} \bigcup_{n>m} h_{n} B\right) & \geq \mu\left((A \cap B) \backslash\left(B \backslash \bigcap_{n=1}^{\infty} h_{n} B\right)\right) \\
& \geq \mu(A \cap B)-\left(\mu(B)-\mu\left(B \cap \bigcap_{n=1}^{\infty} h_{n} B\right)\right) \\
& \geq 2 \varepsilon-(a-(a-\varepsilon))=\varepsilon>0 .
\end{aligned}
$$

We obtain the conclusion of the theorem by Lemma 3.2.

Since each semifinite measure is a localizable extension of itself, we have the following corollary.

Corollary 3.1. Let $\mu$ be invariant and semifinite, and assume that $G$ acts $\mu$ freely. Suppose $L^{1}(\mu)$ has a dense subset of cardinality less than $|G|$. Then each set of positive measure contains a subset nonmeasurable with respect to any invariant extension of $\mu$.

\section{ERGodic MEASURES}

In the proof of the next theorem we will need some lemmas, the first of which is due to Pelc [7, Lemma 3.5] and the second to Bierlein [2, Satz 2B]. For a simple proof of Bierlein's result see [1, Corollary 2].

Lemma 4.2 (Pelc). Let a group $H$ act on a set $A$, and let $\mu$ be a finite $H$ invariant measure on $A$. Assume $A=\bigcup_{i=1}^{n} A_{i}$ and $\mu^{*}\left(A_{i} \cap A_{j}\right)=0$ if $i \neq j$. 
Assume further that for each $h \in H$ and each $i \leq n$ there is $j \leq n$ with $h A_{i}=$ $A_{j}$. Then there exists an $H$-invariant extension of $\mu$ for which $A_{1}, \ldots, A_{n}$ are measurable.

Lemma 4.2 (Bierlein). Let $\left\{A_{i}: i \in N\right\}$ be a countable partition of $A$. Let $\mu$ be a finite measure on $A$. Then there exists an extension of $\mu$ for which $A_{i}$, $i \in N$, are measurable.

Lemma 4.3. Let $m$ be an invariant ergodic measure. Let $A$ be measurable, and let $B$ be measurable of positive measure. Then there exists a countable set $K \subset G$ such that

$$
m(A)=m(A \cap K B)
$$

and

$$
m\left(h^{\prime} B \cap\left(A \backslash \bigcup_{h \in K \backslash\left\{h^{\prime}\right\}} h B\right)\right)>0 \text { for any } h^{\prime} \in K .
$$

Proof. We choose recursively $h_{\alpha}, \alpha$ being an ordinal, until the condition

$$
m\left(h_{\alpha} B \cap\left(A \backslash \bigcup_{\gamma<\alpha} h_{\gamma} B\right)\right)>0
$$

cannot be fulfilled. Let $\bar{\alpha}=\sup \left\{\alpha \leq \omega_{1}: h_{\alpha}\right.$ is defined $\}$. If $\bar{\alpha}<\omega_{1}$, then $m\left(A \backslash \bigcup_{\alpha<\bar{\alpha}} h_{\alpha} B\right)=0$ because $m$ is ergodic, and we get the conclusion putting $K=\left\{h_{\alpha}: h_{\alpha}<\bar{\alpha}\right\}$. If $\bar{\alpha}=\omega_{1}$ then for sufficiently large $\gamma<\omega_{1}$ we have $m(A)=m\left(A \cap \bigcup_{\alpha<\gamma} h_{\alpha} B\right)=\infty$ and $K=\left\{h_{\alpha}: \alpha<\gamma\right\}$ is as required.

Lemma 4.4. Let $A$ be a $\mu$-measurable subset of $X$ such that $\mu(h A \triangle A)=0$ or $\mu(h A \cap A)=0$ for all $h \in G$. Let $H=\{h \in G: \mu(h A \triangle A)=0\}$, and let $\mu_{1}$ be an $H$-invariant extension of $\mu \mid A$ to a $\sigma$-algebra of subsets of $A$. Then there exists an invariant extension $\mu_{2}$ of $\mu$ such that $\mu_{2} \mid A=\mu_{1}$.

Proof. Any element of the invariant $\sigma$-algebra generated by the $\sigma$-algebra of $\mu$-measurable sets and the $\sigma$-algebra of $\mu_{1}$-measurable sets is of the form $\bigcup_{h \in K} h A_{h} \cup B$ for some countable $K \subset G$ with $h_{1} h_{2}^{-1} \notin H$ for $h_{1} \neq h_{2}$, $h_{1}, h_{2} \in K$, where $B$ is $\mu$-measurable and $A_{h} \subset A$ are $\mu_{1}$-measurable. Since $\bigcup_{h \in K} h A_{h} \subset \bigcup_{h \in K} h A$, we can assume that $B \cap \bigcap_{h \in K} h A_{h}=\varnothing$. Moreover, if $h_{1} h_{2}^{-1} \notin H$, we have $\mu\left(h_{1} A \cap h_{2} A\right)=0$. This allows us to put

$$
\mu_{2}\left(\bigcup_{h \in K} h A_{h} \cup B\right)=\sum_{h \in K} \mu_{1}\left(A_{h}\right)+\mu(B) .
$$

It is not difficult to check that $\mu_{2}$ has the desired properties.

In order to formulate the next theorem we have to introduce some new notions. $\Omega \subset X$ is called an atom of a measure $m$ if $m(\Omega)>0$ and $m(A)=0$ or $m(A)=m(\Omega)$ for any $m$-measurable set $A \subset \Omega$. A measure is called atomic if it has an atom and nonatomic if it is not atomic. Now we define a notion which will allow us to divide the class of all atomic invariant ergodic measures into two subclasses. For an invariant measure $m$ and an $m$-measurable set $\Omega$ we put

$$
[\Omega]=\{h \in G: m(h \Omega \triangle \Omega)=0\} .
$$

It is easy to see that $[\Omega]$ is a subgroup of $G$. 
In the following two observations by $\#(A)$ we mean the cardinality of $A$ if $A$ is finite and $\infty$ if $A$ is infinite.

Observation 4.1. Let $m$ be invariant and ergodic, and let $\Omega_{1}, \Omega_{2}$ be two atoms of $m$. Then $\left[\Omega_{1}\right]$ and $\left[\Omega_{2}\right]$ are conjugate; thus, in particular, $\#\left(\left[\Omega_{1}\right]\right)=$ $\#\left(\left[\Omega_{2}\right]\right)$.

Proof. By ergodicity there is $h \in G$ with $m\left(h \Omega_{1} \cap \Omega_{2}\right)>0$, which means $m\left(h \Omega_{1} \cap \Omega_{2}\right)=m\left(\Omega_{1}\right)=m\left(\Omega_{2}\right)$ as $m$ is invariant and $\Omega_{1}, \Omega_{2}$ are atoms. Now it is easy to check that $h\left[\Omega_{1}\right] h^{-1}=\left[\Omega_{2}\right]$.

The above observation indicates that there are basically two types of ergodic atomic measures. We call an ergodic atomic measure a measure of type 1 or of type 2 depending on whether [ $\Omega$ ] is finite or infinite for any (or equivalently some) atom $\Omega$. Ergodic measures of type 1 will turn out to be exceptional from our point of view (see Theorem 3 ). In the next observation we look closer at their nature showing that nontrivial measures of type 1 and counting measures behave in a very similar manner.

Observation 4.2. Let $m$ be a nontrivial ergodic measure of type 1 . Then there exists a positive constant $\alpha \in R$ such that $m(A)=\alpha \#(\{h \in G: m(h \Omega \backslash A)=0\})$ for any atom $\Omega$ and any m-measurable set $A$.

Proof. Let $\Omega$ be any atom. Since $m$ is nontrivial and ergodic, $m(\Omega)<\infty$. Put $H=[\Omega]$. Then $H$ is finite. Put $\alpha=m(\Omega) / \#(H)$. By Observation 1 and its proof $\alpha$ does not depend on $\Omega$. Take any $m$-measurable set $A$. We prove that $m(A)=\alpha \#(\{h \in G: m(h \Omega \backslash A)=0\})$. This is obvious when $m(A)=0$, and when $m(A)=\infty$ it follows immediately from Lemma 4.3 since $m(\Omega)<\infty$. Assume $0<m(A)<\infty$. Applying Lemma 4.3 to $A$ and $\Omega$ and taking into account that $\Omega$ is an atom, we obtain a finite set $K \subset G$ such that $m(A \triangle K \Omega)=0$ and $m\left(h^{\prime} \Omega \cap \bigcup_{h \in K \backslash\left\{h^{\prime}\right\}} h \Omega\right)=0$ for any $h^{\prime} \in K$. This implies that $h_{1} H \cap h_{2} H=\varnothing$ for $h_{1}, h_{2} \in K$ with $h_{1} \neq h_{2}$, and, since $\Omega$ is an atom, $m(h \Omega \backslash A)=0$ if and only if $h \in h^{\prime} H$ for some $h^{\prime} \in K$. We finally get

$$
\begin{aligned}
m(A) & =m\left(\bigcup_{h \in K} h \Omega\right)=\sum_{h \in K} m(h \Omega)=m(\Omega) \#(K)=\alpha \#(H) \#(K) \\
& =\sum_{h \in K} \#(h H)=\alpha \#(\{h \in G: m(h \Omega \backslash A)=0\}) .
\end{aligned}
$$

Since, as is well known (see, e.g., [5, Exercise 59.4, p. 261]), Haar measures on locally compact groups are ergodic, the following theorem provides an abstract version of theorems with topological assumptions (see, e.g., [7, Proposition 2.3]).

Theorem 4.1. Let $m$ be an invariant ergodic measure. Let $\mu$ be its localizable extension. Assume that the action of $G$ is $\mu$-free. Then each set of positive $\mu$ measure contains a subset nonmeasurable with respect to any invariant extension of $\mu$ if and only if $m$ is nonatomic or atomic of type 2 .

Moreover, if $m$ is of type 1 then for each set $D \subset X$ with $\mu^{*}(D)<\infty$ there exists an invariant extension of $\mu$ for which $D$ is measurable.

Proof. First we prove the implication from the right to the left. We have two cases. 
Case 1. $m$ is nonatomic. Let $A$ be such that $\mu(A)>0$. We can find $C$ with $m(C)<\infty$ and $\mu(A \cap C)>0$. By nonatomicity using the standard argument we find $B_{k}$ such that $0<m\left(B_{k}\right)<1 / 2^{k}$. By Lemma 4.3 there are sets $K_{k} \subset G$ such that $m\left(C \backslash \bigcup_{h \in K_{k}} h B_{k}\right)=0$. Since $m(C)>0, \lim _{k \rightarrow \infty}\left|K_{k}\right|=\infty$. Thus $K=\bigcup_{k=1}^{\infty} K_{k}$ is infinite. Let $K=\left\{h_{n}: n \in K\right\}$ with $h_{n} \neq h_{m}$ for $n \neq m$. Let $B=\bigcup_{k=1}^{\infty} B_{k}$. We have $m(B)<\infty$. Notice that

$$
\mu\left(C \backslash \bigcup_{n>m} h_{n} B\right) \leq \inf _{k \in N} \mu\left(C \backslash \bigcup_{n>m} h_{n} B_{k}\right) \leq \inf _{k \in N} m / 2^{k}=0
$$

for each $m \in N$. Thus we obtain

$$
\begin{aligned}
\mu\left(A \cap \bigcap_{m=1}^{\infty} \bigcup_{n>m} h_{n} B\right) & \geq \mu\left(A \cap C \cap \bigcap_{m=1}^{\infty} \bigcup_{n>m} h_{n} B\right) \\
& =\inf _{m \in N} \mu\left(A \cap C \cap \bigcup_{n>m} h_{n} B\right) \\
& \geq \mu(A \cap C)-\sup _{m \in N} \mu\left(C \backslash \bigcup_{n>m} h_{n} B\right)=\mu(A \cap C)>0,
\end{aligned}
$$

and we are done by Lemma 3.2.

Case 2. $m$ is atomic of type 2. Let $\Omega$ be an atom of $m$. Then [ $\Omega$ ] is infinite. Let $\left\{h_{n}: n \in N\right\} \subset[\Omega]$ with $h_{n} \neq h_{m}$ for $n \neq m$. Let $A$ be $\mu$ measurable with $\mu(A)>0$. Find an $m$-measurable set $B$ with $\mu(A \cap B)>0$ and $m(B)<\infty$. By the ergodicity of $m$, using Lemma 4.3 we can find a countable set $K \subset G$ with $m(B \cap K \Omega)=m(B)=\mu(B)>0$. Thus there is $h \in G$ with $\mu(A \cap h \Omega)>0$. We have

$$
\mu\left(h \Omega \cap \bigcap_{m=1}^{\infty} \bigcup_{n>m} h h_{n} \Omega\right)=\mu\left(\Omega \cap \bigcap_{m=1}^{\infty} \bigcup_{n>m} h_{n} \Omega\right)=\mu(\Omega) .
$$

Thus $\mu\left(A \cap \bigcap_{m=1}^{\infty} \bigcup_{n>m} h h_{n} \Omega\right)>0$, and we get the conclusion by Lemma 3.2.

It is clear that the "moreover part" of the theorem is just a stronger form of the implication from the left to the right; thus, we will be done if we prove this part of the theorem only. Assume that $m$ is atomic of type 1 . Let $H=[\Omega]$ for an atom $\Omega$. Then $H$ is finite. Let $\bar{\Omega}=\bigcap_{h \in H} h \Omega$. Then $m(\Omega \backslash \bar{\Omega})=0$ and $h \bar{\Omega}=\bar{\Omega}$ for $h \in H$ and $m(h \bar{\Omega} \cap \bar{\Omega})=0$ for $h \in G \backslash H$. Now we consider the group $H$ as acting on $\bar{\Omega}$. Let $V$ be a Vitali set of $H$ with respect to this action. Since $H$ acts $\mu$-freely, $\mu^{*}\left(h_{1} V \cap h_{2} V\right)=0$ for $h_{1} \neq h_{2}$. Let $\left\{A_{1}, \ldots, A_{n}\right\}=\{h V: h \in H\}$ with $\mu^{*}\left(A_{i} \cap A_{j}\right)=0$ for $i \neq j$. Consider $\mu \mid \bar{\Omega}$. Then the conditions of Lemma 4.1 are fulfilled and we obtain an $H$-invariant extension $\mu_{1}$ of $\mu \mid \bar{\Omega}$ for which $h V, h \in H$, are measurable. By Lemma 4.4 we obtain an invariant measure $\mu_{2}$ with $\mu_{2} \mid \bar{\Omega}=\mu_{1}$ which extends $\mu$. Denote by $\bar{\mu}$ the measure completion of $\mu_{2}$. Obviously, $\bar{\mu}$ is an invariant extension of $\mu$ for which $V$ is measurable.

Let $A$ be a set of finite $\mu$-measure containing $D$. Since $\mu$ is a localizable extension of $m$, an easy argument shows that there is an $m$-measurable set $A^{\prime}$ of $\sigma$-finite measure with $\mu\left(A \backslash A^{\prime}\right)=0$. Lemma 4.3 implies that there is a countable set $K \subset G$ with $m\left(A^{\prime} \backslash K \Omega\right)=0$. Thus $\bar{\mu}(D \backslash K H V)=0$. Put 
$K^{\prime}=K H$. It is enough by Lemma 4.4 to extend $\bar{\mu} \mid V$ to a measure $\mu_{1}$ on $V$ for which $h^{-1} D \cap V, h \in K^{\prime}$, are measurable. (Here the group $\{h \in$ $G: \mu(h V \Delta V)=0\}$ is trivial; thus, we do not have to worry about any invariant properties of $\mu_{1}$.) Let $K^{\prime}=\left\{h_{n}: n \in N\right\}$, and let $D_{\infty}=\bigcap_{k \in N} \bigcup_{n>k} h_{n}^{-1} D \cap V$. Then

$$
D_{\infty} \subset \bigcap_{k \in N} \bigcup_{n>k} h_{n}^{-1} A \cap V .
$$

Notice that if $h_{1}^{-1} h_{2} \in H$ and $h_{1} \neq h_{2}$ then $\mu^{*}\left(h_{1} V \cap h_{2} V\right)=0$ and if $h_{1}^{-1} h_{2} \notin H$ then $h_{1} V \cap h_{2} V \subset h_{1} \Omega \cap h_{2} \Omega$ and $m\left(h_{1} \Omega \cap h_{2} \Omega\right)=0$; whence, in any case $\bar{\mu}\left(h_{1} V \cap h_{2} V\right)=0$ if $h_{1} \neq h_{2}$. Therefore,

$$
\sum_{n=1}^{\infty} \bar{\mu}\left(A \cap h_{n} V\right) \leq \bar{\mu}(A)<\infty .
$$

Thus, as $\bar{\mu}(V)<\infty$, we have

$$
\bar{\mu}\left(\bigcap_{k \in N} \bigcup_{n>k} h_{n}^{-1} A \cap V\right) \leq \inf _{k \in N} \sum_{n>k} \bar{\mu}\left(h_{n}^{-1} A \cap V\right)=\inf _{k \in N} \sum_{n>k} \bar{\mu}\left(A \cap h_{n} V\right)=0 .
$$

Thus $\bar{\mu}\left(D_{\infty}\right)=0$. Moreover, each point in $V \backslash D_{\infty}$ belongs to finitely many sets in $\left\{h_{n}^{-1} D \cap V: n \in N\right\}$; whence, the partition $\left\{A_{n}: n \in N\right\}$ of $V \backslash D_{\infty}$ generated by this family is countable. Hence each $\sigma$-algebra containing $\left\{A_{n}: n \in N\right\}$ and all $\bar{\mu}$-measurable sets also contain $\left\{h_{n}^{-1} D \cap V: n \in N\right\}$. By Lemma 4.2 there is an extension $\mu_{1}$ of $\bar{\mu} \mid V$ for which $A_{n}, n \in N$; whence, also $h_{n}^{-1} D \cap V$, $n \in N$, are measurable.

It is clear that each nontrivial invariant ergodic measure is semifinite. Thus we obtain the following corollary.

Corollary 4.1. Let $\mu$ be a nontrivial nonatomic invariant ergodic measure for which the action of $G$ is $\mu$-free. Then each set of positive measure contains a subset nonmeasurable with respect to any invariant extension of $\mu$.

\section{REFERENCES}

1. A. Ascherl and J. Lehn, Two principles for extending probability measures, Manuscripta Math. 21 (1977), 43-50.

2. D. Bierlein, Über die Fortsetzung von Wahrscheinlichkeitsfeldern, Z. Wahr. Verw. Gebiete 1 (1962), 28-46.

3. R. Engelking, General topology, PWN, Warsaw, 1977.

4. P. Erdös and R. D. Mauldin, The nonexistence of certain invariant measures, Proc. Amer. Math. Soc. 59 (1976), 321-322.

5. P. R. Halmos, Measure theory, Van Nostrand, New York, 1950.

6. A. B. Harazišvili, On some types of invariant measures, Soviet Math. Dokl. 16 (1975), no. 3, 681-684; English transl., Dokl. Akad. Nauk SSSR 222 (1975), no. 3, 538-540.

7. A. Pelc, Invariant measures and ideals on discrete groups, Dissertationes Math. (Rozprawy Mat.) 255 (1986). 
8. C. Ryll-Nardzewski and R. Telgársky, The nonexistence of universal invariant measures, Proc. Amer. Math. Soc. 69 (1978), 240-242.

9. P. Zakrzewski, The existence of universal invariant semiregular measures on groups, Proc. Amer. Math. Soc. 99 (1987), 507-508.

Mathematical Institute, University of Wroclaw, Pl. Grunwaldzki 2/4, 50-384 Wroclaw, Poland

Current address: Department of Mathematics, California Institute of Technology, Pasadena, California 91125 